Cell Research (2002); 12(1):1-7

http://www.cell-research.com

REVIEW

\title{
Telomere and telomerase in oncology
}

JIAO MU*, LI XIN WEI

International Joint Cancer Institute, Second Military Medical University, Shanghai 200433, China

\begin{abstract}
Shortening of the telomeric DNA at the chromosome ends is presumed to limit the lifespan of human cells and elicit a signal for the onset of cellular senescence. To continually proliferate across the senescent checkpoint, cells must restore and preserve telomere length. This can be achieved by telomerase, which has the reverse transcriptase activity. Telomerase activity is negative in human normal somatic cells but can be detected in most tumor cells. The enzyme is proposed to be an essential factor in cell immortalization and cancer progression. In this review we discuss the structure and function of telomere and telomerase and their roles in cell immortalization and oncogenesis. Simultaneously the experimental studies of telomerase assays for cancer detection and diagnosis are reviewed. Finally, we discuss the potential use of inhibitors of telomerase in anti-cancer therapy.
\end{abstract}

Key words: Telomere, telomerase, cancer, telomerase assay, inhibitor.

\section{Telomere and cell replicative senescence}

Telomeres, which are located at the end of chromosome, are crucial to protect chromosome against degeneration, rearrangment and end to end fusion[1]. Human telomeres are tandemly repeated units of the hexanucleotide TTAGGG. The estimated length of telomeric DNA varies from 2 to 20 kilo base pairs, depending on factors such as tissue type and human age. The buck of telomeric DNA is doublestranded, but the end of telomeric DNA consists of 3 ' overhang of single-stranded repeats. Sequencespecific DNA-binding proteins (TRF1 and TRF2) attach to the telomeric DNA repeats. These proteins help maintain telomere stability and regulate telomere length[2].

Owing to the nature of lagging-strand DNA synthesis, traditional DNA polymerases are unable to completely replicate the ends of linear DNA[3]. Incomplete replication leads to the loss of 50-200

* Corresponding author, Tel: 008621-25070891, Fax: 00862125070887, E-mail: mujiao@yahoo.com base pairs of the end of telomeric DNA with each round of DNA replication. Hence, the continual cycles of cell growth and division bring on progressing telomere shortening[4]. Now it is clear that telomere shortening is responsible for inducing the senescent phenotype that results from repeated cell division, but the mechanism how a short telomere induces the senescence is still unknown. Possible mechanism includes the short telomere inducing a DNA damage response, releasing transcriptional regulatory factors from sequestration, and relaxing heterochromatin which can lead to the arrest of cell growth and the cell senescence[5]. Senescence represents a state in which cells no longer proliferate but remain viable for extended periods. The cells which are about to enter the senescent state can be induced to bypass senescence through the introduction of viral oncogenes such as SVA40 Large T antigen (LT) and the HPV E6 or E7 protein. These viral proteins can inactivate p53 and $\mathrm{Rb}$ tumor suppressor pathways, which play a pivotal role in inducing the senescence phenotype. These cells that bypass senescence state continue to proliferate and suffer from further telomere loss. So the shortening 
of telomeres continually progress until the cells enter a second proliferative block named as crisis. After the period most of the cells are destined to die.

\section{Structure and function of telomerase}

One mechanism to restore telomere length is the activation of the specific reverse transcriptase, telomerase. The human catalytic core of telomerase is composed of an RNA component (human telomerase RNA, hTR) which serves as the template for telomere elongation and a protein subunit (human telomerase reverse transcriptase, hTERT) which acts to catalyze the telomere synthesis reaction. So telomerase is an RNA-dependent DNA polymerase which uses its own RNA component as a template to elongate the 3 ' single-strand DNA extension of the chromosome terminus by the synthesis of telomeric repeats. The human telomerase RNA, hTR, contains one and five-six repeats of the template, the sequence 5' -CUAACCCUAAC-3' which is complementary to the telomere repeat. The sequence is complementary to one entire telomeric hexanucleotide and a fraction of adjacent repeats and so constitutes the template region of the molecule [6]. The telomerase complex also comprises hTERTassociated proteins such as Hsp90 and p23, which are required for activity of the enzyme and TLP-1 [7], but the function of these proteins is still unknown. The course of the telomeric DNA elongation by telomerase catalysis is that the 3 ' end of the template region in the RNA molecule anneals to the 3 ' overhang of the telomeric DNA, which generates a base-paired primer required for the reverse transcriptase activity. A new telomeric sequence is synthesized from the 5' to 3' direction and the elongation is followed by a translocating event that repositions the RNA template so that the process can be repeated.

\section{Telomerase and cell immortalization}

Telomere length maintenance seems to be essential in the immortalization process. Since most of human normal somatic cells are telomerase negative, telomere shortening progresses with each cell division. The fact led researchers to propose that telomeres may act as a mitotic clock: once a critically short length is reached, cells encounter a proliferative barrier which may be overcome by the introduc- tion of telomerase activity and the subsequent telomere length maintenance[8]. According to the telomere hypothesis, telomerase reactivation in present cells would permit not only telomere length maintenance but also cellular lifespan extension[9]. In some normal human cell types such as retinal pigmented epithelial cells and fibroblasts, ectopic expression of hTERT can allow these cells to avoid both senescence and the subsequent crisis and obtain immortalization[10], [11]. However, certain cell types require hTERT as well as other factors to achieve cellular immortalization. Partial or complete inactivation of either the $\mathrm{p} 53 / \mathrm{p} 21^{\text {CIP1/WAF1 }}$ or the $\mathrm{pRb} / \mathrm{p} 16^{\mathrm{INK} 4 \mathrm{a}}$ pathway seems to be an important additional requirement for some cell types, such as keratinocytes and mammary epithelial cells, to be immortalized by telomerase reactivation. For example, hTERT can collaborate with viral oncoproteins, such as SV40LT (which inactivates p53 and pRb) or human papillomavirus-16 E7 (which inactivates $\mathrm{pRb}$ ), to escape from senescence [12],[13].

Most normal cells that lack detectable telomer ase activity also lack hTERT expression, whereas hTR and some of the telomerase-associated prote ins can be detected in these cells. These data hav e led to the hypothesis that hTERT expression is the essential factor of the telemerase activity and suggest that regulation of hTERT expression ma $y$ be a key factor for the extension of the cellular $r$ eplicative lifespan and cell immortalization[14], [15]. The mechanism by which telomerase is repr essed in normal somatic tissues while activated in tumor cells appears to include the regulation of th e expression of hTERT, but unfortunately the $\mathrm{m}$ echanism is far from clearness at present. Recen $t$ studies have shown that the hTERT promoter $i$ $\mathrm{s}$ inactive in normal human somatic cells, while b ecomes activated during cell immortalization[16]. Sequence analysis has shown that the hTERT pr omoter contains binding sites for several transcri ption factors, which suggests that hTERT expres sion may be subject to multiple levels of control a nd regulated by different factors in different cell ular context. Experiments have shown that expre ssion of hTERT in both normal human mammar y epithelial cells and normal human fibroblasts is 
activated by c-Myc and the effect contributes to dire ct interaction of c-Myc with the hTERT promoter[1 7]. Treatment of human leukemic cells with anti-se nse pentadecadeoxynucleotides targeted against cMyc mRNA leads to inhibition of telomerase activity [18]. The presence of hTERT transcripts that woul $\mathrm{d}$ result in a truncated or inactivated protein in tum or cell lines indicates that in addition to transcriptio nal activation/repression of the hTERT gene, altern ative splicing can also regulates telomerase activity. Existing evidence of posttranslational regulation of telomerase relates to the involvement of serine/thre onine protein kinases in the phosphorylation of $\mathrm{hT}$ ERT, which is necessary for the activation of the en zyme[19]. There is still evidence shows that some $u$ nidentified repressor genes of hTERT located in th e short arm of chromosome 3 can suppress telomera se activity and the reconstitution of the chromosome can produce permanent growth arrest in breast tum or cells[20]. In an animal model, treatment with an antagonist of growth hormone-releasing hormone d ramatically decreased telomerase activity in xenogr afted U-87MG human glioblastoma cells[21]. All th ese data show the regulation of telomerase activity $i$ $\mathrm{n}$ vitro and in vivo. Further research about the mec hanism of regulation will provide a basis for better $i$ nvestigation and manipulation of telomerase activit $\mathrm{y}$ as a potential therapeutic modality.

\section{Telomerase assay and the diagnosis of can- cer}

The telomerase activity in a variety of normal and malignant cell types by using a sensitive polymerase chain reaction-based method, known as the telomerase repeat amplification protocol (TRAP) assay, suggests that telomerase activity is present in almost all tumor samples but could not be detected in normal tissues, except for germ cells in the ovary and testis[22]. Later TRAP assay results have been reported from thousands of tumor samples and shown that around $85 \%$ of solid tumor samples have detectable telomerase activity[23]. In our experiment, we have identified the telomerase activity in 293 cells and several human liver carcinoma cells respectively by telomerase TRAP-ELISA assay and non-radioisotopic silver staining telomeric repeat amplification protocol[24],[25]. The high sensitivity of the PCR-based TRAP assay has allowed the analysis of minimal tissue samples, such as fineneedle aspirates of the liver, breast and thyroid, cervical smears, oral washings, and urine[26-31]. In some experiments the sensitivity and specificity of the assay in detecting tumor cells has been compared with the gold standard, that is, cytology. Telomerase activity was used to screen bronchial washings and was positive in 18 of 22 patients with primary or metastatic lung cancers, compared with only one of 19 patients without cancer. In contrast, cytology was suggestive of cancer in 14 of 22 patients with lung cancers and 0 of 19 patients without cancer[32]. At least in this study, the sensitivity of TRAP for cancer detection is superior to that of cytology. For detection of bladder carcinoma in urine speciments, telomerase is proved far more sensitive than cytology or other available screenings. In two independent studies, the sensitivity of telomerase was $85 \%$ and $70 \%$, compared with $51 \%$ and $44 \%$ for cytology [33],[34].

However, the use of telomerase activity assay as an indication of cancer should be separately evaluated for each type of tumors and tissues. For example, although the frequency of telomerase activity in tumor tissue samples is overall as high as $90 \%$, activity is present in only $50 \%$ of glioblastoma and retinoblastoma samples, and activity is even rarely found in meningiomas and astrocytomas[35], [36]. Furthermore the high false-positive rates of the telomerase activity assay have been shown in some experiments. For example, $34 \%$ of patients tested in one study had telomerase-positive urine, predominantly from patients with cystitis.

The distribution of pattern of telomerase activity in some human tissues correlates well with the hTERT mRNA which is specifically upregulated in human tumor cell lines. However, hTR and telomerase-associated protein is expressed in many telomerase-negative tissues and cell lines. Thus the expression of hTERT may be the specific marker of the cancer cells. Wataru Y et al[37] adopted the immunohistochemical assay to detect the expression of hTERT protein in human gastric carcimomas. The result showed that the expression of hTERT protein was strongly positive in the nuclei of the tumor cells of all carcinoma tissues, while the expression of hTERT in non-neoplastic mucosal cells as well as stromal elements (except lymphocytes) was weak or 
negative. According to the findings, researchers suggested that increased hTERT expression associated with telomerase activity may serve as a novel marker for the diagnosis of stomach cancer.Hiyama et al[38] have found that in a smaller series of neuroblastoma (35 cases) hTERT mRNA expressed in all 13 tumors with high telomerase activity and in five of $22 \mathrm{tu}$ mors with low or undetectable telomerase activity. Poreboma C. et al[39] proposed that immunohistochemical detection of hTERT by anti-hTERT antibody-reactive protein in tissue sections may be an indicator of telomerase activity in the tumor cells. Additionally, for retrospective studies on archived materials, RT-PCR to detect hTERT mRNA expression from paraffin-embedded tissues may be another option for indirect assessment of telomerase activity. However, they also suggested that on the one hand RT-PCR from paraffin-embedded tissue-derived RNA may not always allow accurate quantification of gene expression levels, which is an important limitation of directly deducing telomerase activity levels from hTERT expression, and on the other hand, prospective studies with larger case numbers should be performed to further evaluate the prognostic impact of hTERT RNA or protein detection in paraffin samples.

In conclusion, although there is not yet a diagnostic indication where telomerase assays have shown a clear clinical benefit, there may be settings in which telomerase assays could be useful, for example, as adjuncts to cytology. The use of telomerase assays in cancer diagnosis remains an area of further investigation. At the same time, the detection of the expression of hTERT mRNA or protein may be the better complement for telomerase assay in the tumor cells.

\section{Telomerase as a target for anti-cancer therapy}

The evidence of a strong correlation between telomerase activation, cellular immortalization, and oncogenesis has raised the possibility that telomerase inhibitors might be useful agents that could selectively kill cancer cells while sparing normal cells. Studies of telomerase inactivation in human cells shed some light on these possibilities. The desired effect of telomerase inhibition would be to shorten telomeres to critical lengths, causing replicative se- nescence and preferably cell death due to irreparable chromosome damage. These objectives are feasible because the majority of tumors have shorter telomeres and higher proliferation rates than normal replicative cell populations[40], thus inhibition of telomerase activity may only be necessary over a few cellular divisions to attain lethal erosion of telomere. In contrast, the longer telomeres of stem cells and germ line cells should not lose their chromosomal protective function during therapy.

Antisense technology utilizes single-stranded DNAs or RNAs that are complementary to a singlestranded target region, which is usually RNA. Feng et al[6] used antisense oligonucleotides to identify the RNA of human telomerase and reported its cloning and sequencing. When the antisense oligonucleotides to the first 185 nucleotides of the hTR was introduced into HeLa cells, 33 of 41 antisense RNAexpressing clones underwent crisis at 23-26th doublings. Later, an interesting type of antisense agent, peptide nucleic acid (PNA) is produced. This is a DNA mimic in which the entire deoxyribose phosphodiester backbone is replaced by $\mathrm{N}-(2-$ aminoethyl) glycine units. PNA is able to form double helical complexes with single-stranded DNA or RNA and is highly resistant to degradation by protease and nuclease[41]. Norton et al[42] assessed the effect of a series of PNA oligomers of varying length representing antisense polarity to the template region of human telomerase RNA in human primary breast epithelial cell line HME50-5. They found that the most effective oligomers was a 13 mer complementary to nucleotides 48-60 (the template region is from 48-56) Kondo et al[43] studied the effect of a 19-mer antisense oligonucleotide linked to a 2, 5oligoadenylate against human telomerase RNA in human malignant glioma cell lines. This unconventional oligonucleotide activates the endoribonuclease, RNAase L. Using the specific antisense oligonucleotides, the researchers found that the conjugate reduced tumor cell growth both in vitro and in a nude mouse xenograft. They also found that such a compound could reduce the viability of prostate cancer cells in vitro within 6 days and suppressed the growth of subcutaneous tumors in nude mice, both via the induction of apoptosis.

The ribozyme technology is a modification and extension of the antisense technology. The hammer- 
head ribozyme can bind to its target RNA sequence and induce a specific chemical cleavage reaction. Yokoyan et al[44] designed a hammerhead ribozyme which can specifically cleave the 44-46 GUC sequence in human telomerase RNA. When the ribozyme was transfected into endometrial cancer cells AN3CA, it inhibited telomerase activity in some clones, but not others. In AN3CA cells, the greater the expression of the ribozyme, the lower the concentration of telomerase RNA. The clones showing the greatest reduction in telomerase RNA manifested telomere shortening.

Some anti-cancer chemotherapeutic agents have been identified to have the ability to inhibit the telomerase activity. Burger et al[45] founded that when a testicular cancer cell line was cultured with cisplatin(a chemotherapeutic agent) telomerase activity was inhibited. The possible mechanism was that cisplatin cross-linked DNA between guanines. $\mathrm{Ku}$ et al[46] found that protein kinase $\mathrm{C}$ inhibitors decreased telomerase activity when assayed in cell extracts after they had been added to cultured human nasopharyngeal cancer cells. Among pKC inhibitors, $\mathrm{H}-7$ and bisindolymaleimide were the most active one. Sharma et al[47] showed that telomerase activity was inhibited when dimethyl sulfoxide (DMSO) was used to arrest the proliferation of Burkitt lympoma cells. When proliferation was restored by removing DMSO, both proliferation and telomerase activity were promptly restored.

Some researchers have found that the wild-type p53 and Rb gene transfected by a recombinant adenoviral vector repressed telomerase activity and later arrested the cell growth or led to the cell apoptosis. Kanaya et al[48] suggested that the inhibition of telomerase by p53 seemed to be mediated by an effect on the hTERT promoter, possibly at the binding sites for the transcription factor Sp1.

One of the major limitations of targeting telomerase in malignancies is that telomeres must shorten considerably before the cells enter the state of senescence. This would take many cell generations, perhaps more than that would be required for the cancer to be lethal. One measure to possibly overcome the difficulty is the use of telomerase as a tumor antigen. Partial peptides from the telomerase reverse transcriptase hTERT were shown to elicit a cytotoxic T-lymphocyte response which could lyse hTERT positive tumor cell lines [49]. However, this approach has not yet been identified in vivo, and the effects on normal telomerasepositive cells with the antigen remain unknown. Nevertheless, as a novel and potential anti-cancer way, such approaches deserve to be further investigated.

These results are apparently interesting and suggest new possibilities for the treatment of cancer. However, we must remember several points as follows. First, some malignant tumors, around 10\%$15 \%$, do not express telomerase activity and hence these tumor cell lines may use a method other than telomerase activation to extend telomeres[50]. ]For these tumors, the inhibitor of the telomerase activity may be unuseful. Second, the inhibitors worked best in cultured tumor cells when telomeres were shortest, but little is known about the length of telomeres in primary human tumors. Third, it is argued that an effective inhibitor of telomerase might impair the function of normal cells, which have low levels of telomerase activity[51]. Even though the therapeutic potential of telomerase inhibitors may be limited by these considerations, further investigation of this approach is certainly worthwhile.

\section{Summary}

The telomere hypothesis of cancer cell immortalization-telomere shortening limits the lifespan of telomerase-negative normal cells, whereas telomerase activation in cancer cells extends their lifespan. Telomerase activation has been shown to extend the lifespan of normal human somatic cells in culture and can contribute to experimental tumorigenesis. On the other hand, telomerase activity may not be the only mechanism for restoring eroded telomere sequences although the enzyme appears to be a key factor in the maintenance of telomeres. The detection of telomerase in human tissue samples may also be one of the best markers of cancer with a broad range of tumor types, but the drawback of higher false-positive rate must be overcome. Telomeric integrity is essential for the stability of chromosomes and so telomerase is a target for therapeutic intervention. In the long run, the potential uses of telomerase inhibitors for cancer treatment remain promising but await the development of suitable inhibitors for further testing. We 
anxiously anticipate the exciting developments that the next decade will bring.

\section{REFERENCES}

[1] Sandell LL, Zakian VA. Loss of a yeast telomere: arrest, recovery, and chromosome loss. Cell 1993; 75:729-39.

[2] Broccoli D, Smogorzewska A, Chong L,de Lang T. Human telomeres contain two distinct Myb-related proteins, TRF1 and TRF2. Nat Genet 1997; 17:231-5.

[3] Lingner J, Cooper JP, Cech TR. Telomerase and DNA end replication:no longer a lagging strand problem? Science 1995; 269:1533-4.

[4] Counter CM, A vilion AA, LeFeuvre CE, et al. Telomere shortening associated with chromosome instabiliby is arrested in immortal cells which express telomerase activity. EMBO J 1992; 11:1921-9.

[5] Campisi J.The biology of replicative senescence. Europ J Canc 1997; 33:703-9. Feng J, Funk WD, Wang SS, et al. The RNA component of human telomerase. Science 1995; 269:1236-41.

[6] Feng J, Funk WD, Wang SS, et al. The RNA component of human telomerase. Science 1995; 269:1236-41.

[7] Harrington L, Mcphail T, Mar V, et al.A mammalian telomerase-associated protein Science 1997; 275:973-7.

[8] Harley CB. Telomere loss: mitotic clock or genetic time bomb? Mutat Res 1991; 256:271-82.

[9] Harly CB. Telomeres and aging. Cold Spring Harbor: Cold Spring Harber Laboratory Press 1995; 247-63.

[10] Bodnar AGM, Ouellettee M, Frolkis M, Holt SE. Extension of life-span by introduction of telomerase into normal human cells. Science 1998; 279:349-52.

[11] Vaziri H, Benchimol S. Reconstitution of telomerase activity in normal human cells leads to elongation of telomeres and extended replication life span. Curr Biol 1998; 8:279-82.

[12] Morales CP, Holt SE, Ouellette M, et al. Absence of cancer-associated changes in human fibroblasts immortalized with telomerase. Nat Genet 1999; 21:115-8.

[13] Kiyono T, Foster SA, Koop JI, et al. Both Rb/p16INK4a inactivation and telomerase activation are required to immortalize human epithelial cells. Nature 1998; 396: 84-8.

[14] Avilion AA, Piatyszek MA, Gupta J, et al. Human telomerase RNA and telomerase activity in immortal cell lines and tumor tissues. Cancer Res 1996; 56:64550 .

[15] Nakamura TM, Morin GB, Chapman KB, et al. Telomerase catalytic subunit homologs from fission yeast and human. Science 1997; 277:955-9.

[16] Cong YS, Wen J, Bacchetti S. The human telomerase catalytic subunit hTERT: organization of the gene and characterization of the promoter. Hum Mol Genet 1999; 8:137-42.

[17] Horikawa I, Cable PL, Afshari C, Barrett JC. Cloning and characterization of the promoter region of human telomerase reverse transcriptase gene. Cancer Res 1999; 59:826-80.
[18] Fujimoto K, Takahashi M.Telomerase activity in human leukemic cell lines is inhibited by antisense pentadecadeoxynucleotides targeted against c-myc mRNA. Biochem. Biophys Res Commun. 1997; 241:77581.

[19] Li H, Zhao L,Yang Z, et al. Telomerase is controlled by protein kinase $\mathrm{C}$ alpha in human breast cancer cells. J Biol Chem 1998; 273:33436-42.

[20] Cuthvert AP, Bond J, Trott DA, et al. Telomerase repressor sequences on chromosome 3 and induction of permanent growth arrest in human breast cancer cells. J Natl Cancer Inst 1999; 91:37-45.

[21] Kiares H, Schaly AV. D ecrease in telomerase activity in U-87MG human glioblastomas after treatment with an antagonist of growth hormone-releasing hormone. Proc Natl Acad Sci USA 1999; 96:226-31.

[22] Kim NW, Piatyszek MA, Prowse KR, et al. Specific association of human telomerase activity with immortal cells and cancer. Science 1994; 266:2011-5.

[23] Shay JW, Bacchetti S. A survey of telomerase activity in human cancer. Eur J Cancer 1997; 33:787-91.

[24] Wei Lixin, Wu Mengchao, Guo Yajun, et al. Detection of human telomerase activity by telomerase TRAP-ELISA assay. Chinese Journal of Cancer Research 1997; 9(4): 277-80.

[25] Wei Lixin, Guo Yajun, Wu Mengchao, et al. Detection of telomerase activity in human cells with telomeric repeat amplification protocol by silver staining. Med Cell PLA 1998; 13(2):140-2.

[26] Zhang Ru Gang, Xing Wang Wang, et al. Using a nonradioisotopic,quantitative TRAP-based method detecting telomerase activities in human hepatoma cells. Cell Research 2000; 10(1):71-8.

[27] Sugino T, Yoshida K, Bolodeoku J, et al. Telomerase activity in human breast cancer and benign breast lesions: diagnostic applications in clinical specimens,including fine needle aspirates. Int $\mathrm{J}$ Cancer 1996; 69:301-6.

[28] Aogi K, Kitahara K, Buley I, et al. Telomerase activity in lesions of the thyroid:application to diagnosis of clinical samples including fine-needle aspirates. Clin Cancer Res 1998; 4:1965-70.

[29] Gorham H, Yoshida K, Sugino T, et al. Telomerase activity in human gunaecological malignancies. J Clin Pathol 1997; 50:501-4.

[30] Duggan BD, Wan M, Yu MC, et al. Dectection of ovarian cancer cells: comparison of a telomerase assay and cytologic examination. J Natl Cancer Inst 1998; 90:238-42.

[31] Kinoshita H, Ogawa O, Kakehi Y, et al. Detection of telomerase activity in exfoliated cells in urine from patients with bladder cancer. J Natl Cancer Inst 1997; 89: 724-30.

[32] Yahata N, Ohyashiki K, Ohyashiki JH, et al. Telomerase activity lung cancer cells obstained from bronchial washings. J Natl Cancer Inst 1998; 90:684-90.

[33] Kavaler E, Landman J, Chang Y, et al. Detection human bladder carcinoma cells in voided urine samples by assaying for the presence of telomerase activity. Cancer 1998; 82:708-14.

[34] Ramakumar S, Bhuiyan J, Besse JA, et al. Comparison of 
screening methods in the detection of bladder cancer. J Urol 1999; 161:394.

[35] Gupta J, Han LP, Wang P, et al. Development of retinoblastoma in the absence of telomerase activity. J Natl Cancer Inst1996; 88:1152-7.

[36] Langford LA, Piatyszek MA, Xu R, et al. Telomerase activity in human brain tumours. Lancet 1995; 346: 1267-8.

[37] Wataru Yasui, Hidetoshi Tahara, Eiji Tahara, et al. Expression of telomerase catalytic compontent, telomerase reverse transcriptase, in human gastric carcinomas. Jpn J Cancer Res 1998; 89:1099-103.

[38] Hiyama E, Hiyama K, Yokoyama T, et al. Rapid detection of MYCN gene amplification and telomerase expression in neuroblastoma. Clin Cancer Res 1999; 5:601-9.

[39] Poreboma C, Scheel C, Hero B et al. Telomerase activity and telomerase subunit gene expression patterns in neuroblastoma:a molecular and immunohistochemical study establishing prognostic tools for fresh-frozen and paraffin-embedded tissues. J of Clin Oncol 2000; 18(13): 2582-92.

[40] Bacchetti S.Telomere maintenance in tumour cells. Cancer Surv 1996; 28:197-216.

[41] Demidov VV, Potaman VN, Frank-Kamenetskii MD, Egholm M, et al. Stability of peptide nucleic acids in human serum and cellular extracts. Biochem. Pharmaco 1994; 48:1310-3.

[42] Norton JC, Piatyszek MA, Wright WE, Shay JW, Corey DR. Inhibition of human telomerase activity by peptide nucleic acids. Nature Biotechnol 1996; 14:615-9.

[43] Kondo S, Kondo S, Tanaka Y, et al. Targeted therapy of human malignant glioma in a mouse model by $2,5-\mathrm{A}$ antisense directed against telomerase RNA. Oncogene
1998; 16:3323-30.

[44] Yokoyama Y,Takahashi Y, Shinohara A, et al. Attenuation of telomerase activity by a hammerhead ribozyme targeting the template region of telomerase RNA in endometrial carcinoma cells. Cancer Res 1998; 58:540610.

[45] Burger AM, Double JA, Newell DR. Inhibition of telomerase activity by cisplatin in human testicular cancer cells. Europ J Cancer 1997; 33:638-44.

[46] Ku WC, Cheng AJ, Wang TCV. Inhibition of telomerase activity by PIC inhibitors in human nasopharyngeal cancer cells in culture. Biochem Biophys Res Communs 1997; 241:730-6.

[47] Sharma S, Raymond E, Soda H, Izbicka E, et al. DMSO causes a reversible inhibition of telomerase activity in a Burkitt lymphoma cell line. Leukemia Res 1998; 22: 663-70.

[48] Kanaya T, Kyo S, Hamada K, et al. Adenoviral expression of p53 represses telomerase activity througy downregulation of human telomerase reverse transcriptase transcription. Clin Cancer Res 2000; 6:1239-47.

[49] Vonderheide RH, Hahn WC, Schultze JL, et al. The telomerase catalytic subunit is a widely expressed tumor-associated antigen recognized by cytotoxic $\mathrm{T}$ lymphocytes. Immunity 1999; 10:673-9.

[50] Byran TM, Englezou A, Gupta J, et al. Telomere elongation in immortal human cells without detectable telomerase activity. EMBO J 1995; 14:4240-8.

[51] Morin GB. Is telomerase a universal cancer target? J Natl Cancer Inst 1995;s:859-60. 